\title{
Filmpädagogik
}

Zurück zur Übersicht über den

Themenschwerpunkt

\section{Spielfilmarbeit mit jugendlichen Strafgefangenen - Film- und Fernsehkompetenz im Knast}

Ein medienpädagogisches Forschungsprojekt zur Methodenrevision traditioneller Medienarbeit und zur Gewaltreflexion durch aktive Film- und Fernsehkompetenz

\section{REINHARD NOLLE}

Film- und Fernsehkompetenz, ein erweiterter Ansatz zur Medienkompetenz

Vor etwa zwanzig Jahren hat sich nicht nur die IT-Welt verändert, mit ihr hat sich auch die soziale Welt außerhalb der Arbeit erheblich gewandelt. Das Privatfernsehen hat seit 1984 Schritt für Schritt unsere Freizeit erobert. Das Genre Spielfilm sowie Musikclips sind mit größtem Abstand $\mathrm{zu}$ allen anderen Genres und Formaten im Fernsehen die Kategorien mit der größten Nutzungsdauer vor dem Fernseher von 215 Min. täglich bei Kindern und Jugendlichen (SevenOne Media, München 2004). Das bedeutet dreieinhalb Stunden täglich oder eineinhalb Tage pro Woche. Es bedeutet bezogen auf ein Menschenleben fast dreizehn Jahre ununterbrochenen Fernsehens. Dazu kommen noch der Kino- und Videokonsum. Es gibt keine Institution in unserer Gesellschaft, die kontinuierlich so viele Menschen dazu bringt, sich so lange Zeit zur selben Stunde mit der gleichen Sache zu beschäftigen. Es gibt nichts, was mit dieser Intensität so vielen Menschen so einheitliche Werte und Moralvorstellungen vermittelt wie das Fernseh- und Videoprogramm. Das Fernsehen ist ein Supermarkt sozialer Images und Botschaften mit eingeschränkter Gefühlwahrnehmung mit Schwarz-weiß- und Gut-böse-Werten und -Ansichten. Fernsehen ist ein gewaltiger Lieferant mit immer neuen Sonderangeboten für die Fast-Food-Kultur.

Die Fragen, wie man sich dieser übermächtigen ökonomischen und mainstreamigen Macht gegenüber stellt und welche Methoden einer emanzipativen Medienpädagogik sich diesen Anforderungen gewachsen sehen, sollten der Forschungsfokus in diesem praxisorientierten Medienprojekt sein.
Es liegt auf der Hand, dass eine Methodenforschung, die die Ausbildung einer modernen Film- und Fernsehkompetenz zum Ziel hat, weniger auf anwendungs-, bedien- und produktionsorientierte Medienkompetenz ausgerichtet ist. Sie konzentriert sich auf ein Kompetenzziel, das sich einerseits an dem Genre Spielfilm orientiert und andererseits auf metatheoretische Reflexionsprozesse ausgerichtet ist sowie auf die Kompetenz zur Bearbeitung einer eigenen Dialog- und Selbstdarstellungsdramaturgie.

\section{Zielgruppe und Kontext}

In der Justizvollzugsanstalt Wiesbaden werden seit Mai 2001 mit einer Gruppe von 10 jugendlichen Strafgefangenen (Alter: 19 bis 24 Jahre; Haftzeit: 3 bis $91 \frac{1}{2}$ Jahre) Medienworkshops in prozessorientierter Spielfilmarbeit im Abstand von vier bis fünf Wochen durchgeführt. Die Gruppe absolviert jeweils sechs bis acht aufeinander folgende Workshops, um neue soziale und empathische Erfahrungen langfristig zu festigen. In dieser Zeit entstehen Kurzspielfilme auf der Grundlage selbst erlebter und selbst aufgeschriebener Geschichten der Gefangenen zu vorgegebenen Themen, wie Gewalt, Drogen, Mitgefühl, Stolz, Beziehungen etc., die auf den biografischen Alltagserfahrungen und Taten der Teilnehmer basieren.

Die Tathintergründe der Strafgefangenen sind Mord, Raub, Handel und Konsum von Drogen, Körperverletzung, Einbruch. Bei fast allen Taten spielen Drogen und Alkohol eine relevante Rolle. Ziel der pädagogischtherapeutischen Arbeit ist die Veränderung der persönlichen Einstellungen, der eigenen Wertehaltung und des Sozial- und Empathieverhaltens. Die jugendlichen Gefangenen arbeiten acht Tage lang ohne Pause in einem sehr intensiven Teamprozess, der unter Zeit- und Produktionsdruck steht, da sie am Ende der etwa 80 Stunden Teamarbeit vor 100 Mitgefangenen der JVA den fertigen Spielfilm vorführen und diskutieren müssen.

Neben dem technischen Entstehungsprozess der Kurzspielfilme, an dem die Teilnehmer in Assistenzfunktionen beteiligt sind, sind es vor allem die Bereiche Dramaturgie, Inszenierung, Regie und der Zwang 
zu Teamarbeit, Reflexion und Disziplin, in denen sie lernen. Sie lernen, wie die eigene Geschichte in eine gemeinsame Geschichte zu integrieren ist. Sie lernen, dass alles im Spielfilm inszeniert ist, dass es keine Zufälle gibt, dass sog. Reality-Shows keine Realität abbilden, sondern die Reality inszeniert wird.

Sie erfahren die Notwendigkeit zur Inszenierung, die Abhängigkeit von Dialog, MimikGestik, Bewegung des Körpers, von Maske und Licht, von Bildgestaltung, Rhythmus, Schnittdramaturgie und dem Zusammenspiel auf ein Gesamtwerk, das sie auf der Metaebene immer im Blick haben. Sie erfahren, dass sie dieses Ziel beeinflussen können, durch die Qualität der eigenen Geschichte, die Qualität der Dialoge und die Qualität der schauspielerischen Ausgestaltung der Rolle in ,ihrem“ Film, der Qualität des eigenen sozialen und empathischen Verhaltens. Die Teilnehmer lernen, dass man selbst tagtäglich viele parallele Geschichten sieht, erlebt oder an ihnen beteiligt ist. Dann lernen sie, wie man Geschichten in Parallelmontagen konstruiert.

In der medienpädagogischen Arbeit mit jugendlichen Strafgefangenen sollten diese Aspekte eines revidierten medienpädagogischen Arbeitsansatzes erprobt sowie didaktische und methodische Fragestellungen thematisiert und durchgespielt werden. Die medienpädagogische Arbeit sollte eine veränderte Selbstreflexionsfähigkeit der Teilnehmer bewirken, auf eine Veränderung der persönlichen Einstellungen abzielen sowie soziale und interaktionelle Kompetenzen fördern. Am Schluss des Projektes, nach ca. 1 Jahr und $6-8$ Spielfilmworkshops pro Gruppe, sollte bei den Teilnehmern die Fähigkeit zur Mentalisierung erkennbar sein: die Fähigkeit, die eigene mentale Verfassung in Zusammenhang mit der mentalen Verfassung anderer Personen zu bringen. Sozialkompetenz und Empathie sind einerseits wesentliche Elemente einer Filmund Fernsehkompetenz und andererseits psychosoziale Bedingungen für die Resozialisierung.

\section{Übergeordnete Ziele}

Die Einsicht in Tat und Strafe, die Reflexion der eigenen Einstellungen und des eigenen Handelns sind die übergeordneten Ziele der medienpädagogischen Arbeit mit jugendlichen Strafgefangenen. Es ist der Anstoß zur Reflexion ihrer Kindheits- und Alltagserfahrungen, ihrer kriminellen Taten, ihres Alltags in der Haft. Die Fähigkeit zur Reflexion, die Einsicht und Bewertung eigenen Handelns ist eine wesentliche Voraussetzung zur Veränderung persönlicher Einstellungen und Ziele und zur Veränderung eigener Wertehaltung und spezifischer Identitätsmuster. Konstitutiv für unterschiedliche Aushandlungsprozesse unterei- nander in der Gruppe ist die Entwicklung und Förderung sozialer und kommunikativer Kompetenz mit dem Ziel einer rücksichtsvollen Diskussionskultur. Das geht nicht ohne eine demokratische Kommunikations- und Alltagskultur und nicht ohne eine selbstverständliche Kultur der Anerkennung und Zuwendung. Im Kontext der oft traumatischen Kindheits- und Jugenderfahrungen der jugendlichen Gefangenen muss das Projekt sich bei den Teilnehmern erst einmal für eine Bereitschaft zum Aufbau von Normalität und friedlichem Zusammenleben in toleranter rücksichtsvoller Gruppenatmosphäre einsetzen. Wichtig sind dabei grundlegende friedliche, in ihrer Idealform unterstützende, mitfühlende Orientierungen zur Lösung von Gruppen- oder individuellen Konflikten und Ereignissen, die eine immer wiederkehrende Verankerung auf das gemeinsame Ziel fokussieren: das Gefühl „sicherer“ Gemeinsamkeit, aufgehoben sein, sich zeigen zu dürfen mit seinen Gefühlen und peinlicher Vergangenheit und den Leiden emotionaler Verlassenheit in einer Strafanstalt.

Die Struktur der Gruppe und ihre inneren Regeln (Schutzraum für die Teilnehmer; außerhalb des Raums wird nichts weitererzählt) fördern stabilisierende Erklärungs- und Verhandlungsprozesse zwischen den Teilnehmern über ihre Alltagserfahrungen, Familiengeschichten, Taten, persönlichen Einstellungen und Wertehaltungen. Diese Prozesse, die die verschiedenen Phasen des Spielfilmprojektes begleiten, von der thematischen Diskussion der eigenen und der anderen Geschichten über den filmischen Produktionsprozess, in dem die Teilnehmer vor der Kamera stehen, bis zur Präsentation des fertigen Spielfilms nach acht Tagen vor 100 Mitgefangenen, fördern das Gefühl von Gemeinsamkeit, Toleranz, sozialem Verhalten und Empathie zueinander. Sie basieren in ihrem Kern auf metatheoretischen Reflexionsprozessen und haben die persönlichen Themen, individuellen Erfahrungen, Interessen und Einstellungen der Teilnehmer zum Schwerpunkt. Diese Prozesse in Gang zu setzen und Einstellungen zu verändern ist das wichtigste Ziel des Projektes.

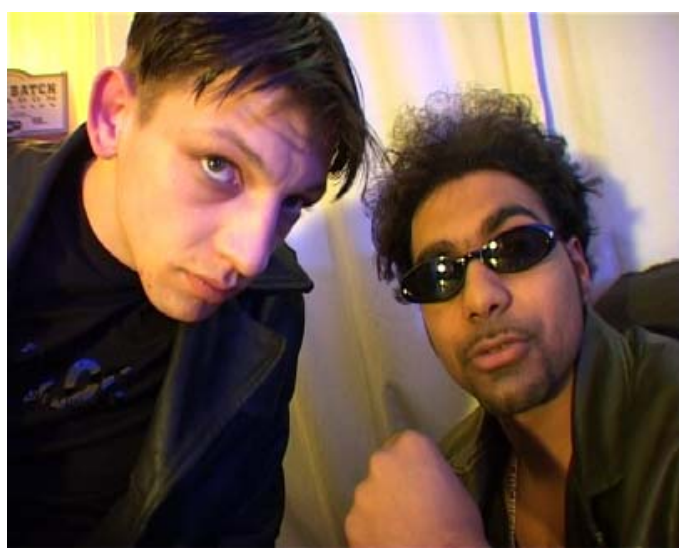


In einem Arbeitsprozess, an dem einerseits die Forschenden und andererseits die Gefangenengruppe als Personen mit ihrem Erleben, ihren Fühl- und Denkmustern beteiligt sind, werden die subjektiven Erfahrungen, Gefühle, Erinnerungen und persönlichen Standpunkte aller an diesen Spielfilmprojekten Beteiligten aufgespürt. Sie werden reflektiert, um eine Bewusstheit der Prozesse und die Bewusstheit von Veränderung, das Überprüfen und vorsichtige Annehmen neuer Wertehaltungen zu erzielen. Dies setzt ein hohes $\mathrm{Maß}$ an Reflexivität, gegenseitigem Respekt und Mitgefühl voraus und gründet sich auf gemeinsame Lernprozesse, die die Teilnehmer durchlaufen auf ihrem Weg zu einem gemeinsamen Ziel, einen kreativen Spielfilm zu erstellen, in dem sie, oft sehr verfremdet, ihre eigenen Rollen spielen.

Im Laufe der Workshops verändert sich wahrnehmbar ihr Verhalten untereinander. Langsam entwickeln sich unter Einzelnen mitfühlende, rücksichtsvolle Unterstützungen bei den schier endlosen Gesprächen, Schauspielproben und manchmal über 20 erfolglosen Versuchen für einen Dialog mit einem Partner während des Drehens. Nach acht Tagen und 80 Stunden gemeinsamer Arbeit warten 100 Mitgefangene, die den Film sehen wollen: ein Erwartungs- und Leistungsdruck an die Filmgruppe, aber auch an die Teamergruppe der Universität, dem sich niemand wirklich entziehen kann.

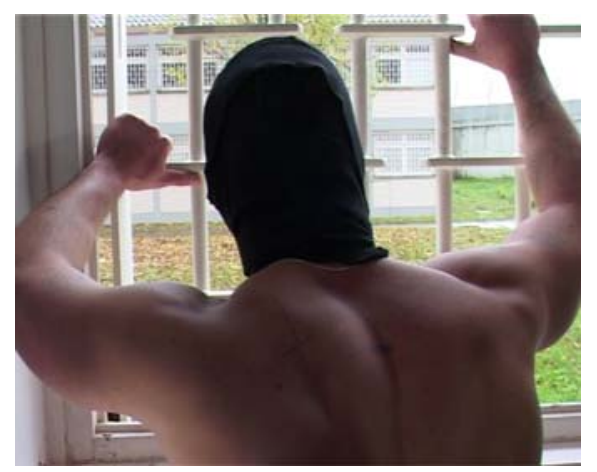

Die Kurzspielfilme mit Laufzeiten bis zu 14 Minuten basieren ausschließlich auf den Alltags-, Kindheits-, Familien- und Taterfahrungen der jugendlichen Straf- und Gewalttäter. Zu vorgegebenen Schwerpunktthemen, wie Gewalt, Rassismus, Beziehungen, Egoismus, Mitgefühl, Toleranz, Eitelkeit, etc., schreiben die Teilnehmer selbst erlebte Geschichten auf. Die Geschichten werden zu einer gemeinsamen Kerngeschichte nach einer in dieser Arbeit entwickelten Methode zusammengefasst. Die gemeinsame Geschichte hat mindestens so viele Rollen, wie es Teilnehmer gibt. In geheimer Wahl werden die Rollen auf einem Rollenplan vorgeschlagen. Die Rollenpläne werden von den Teamleitern ausgewertet. Steht die mit Spannung erwartete Rollenverteilung fest, werden die Dialoge jeweils von den „Schauspielern“, die auch später gemeinsam vor der Kamera spielen werden, in einem gemeinsamen Diskussionsprozess entwickelt und aufgeschrieben. Im nächsten Schritt werden die Dialoge in die gemeinsame Kerngeschichte eingearbeitet. In dieser Arbeitsphase zeigt sich, wie weit die einzelnen Teilnehmer gelernt haben, miteinander umzugehen, bemüht sind, sich gegenseitig zu helfen und ,gemeinsame" Dialoge verschriftlichen können. Das fertige Drehbuch wird in verteilten Rollen gelesen, dabei wird ein bis zwei Mal die Stoppzeit genommen, um die ungefähre Filmlänge zu festzustellen.

\section{Prozessorientierte Spielfilmarbeit}

Die wichtigsten Eckpunkte der prozessorientierten Spielfilmarbeit sind:

- An Alltagserfahrungen der Teilnehmer anknüpfen: Jeder Teilnehmer schreibt seine eigene selbst erlebte Geschichte, keine adaptierten Themen aus dem Fernsehen, keine Fantasiegeschichten.

- Aus den einzelnen Schlüsselworten und Handlungsfragmenten wird eine gemeinsame Geschichte entwickelt. Hinzugefügt werden fiktive Elemente als dramaturgische Mittel, um Rhythmus und Spannung zu erzeugen. Charaktere können verändert werden.

- Jeder spielt mit und schreibt seine eigenen Dialoge mit seinem Film-Partner.

- Identifikation ist wichtig. Jeder Teilnehmer muss sich in einem Teil der gemeinsamen Geschichte wiederfinden.

- Die Sozialarbeiter, Lehrer und Mitarbeiter des Medienteams sind verantwortlich für die technisch dramaturgische Qualität des Films. Kamera, Ton und Bildschnitt liegt in den Händen des Medienteams.

- Die Arbeit erfolgt in Zeitblöcken. Ideal sind acht bis zwölf Tage an einem Stück.

- Die Teilnehmer sind verantwortlich für ihre Geschichte, ihre Dialoge, Schauspiel, Präsentation und Diskussion. Die Betreuer helfen dramaturgisch beim Drehbuch.

- Zu Beginn des Projektes muss der Präsentationstermin (Ort, Zeit) festgelegt werden, Zuschauer und Presse sind einzuladen. Dadurch entsteht Zeit- und Produktionsdruck für die Gruppe. Der Ernstcharakter wird spürbar.

Die technisch dramaturgische Umsetzung des Drehbuchs in einen Spielfilm - Regie, Kamera, Ton, Licht - leisten die Mitarbeiter der MedienWerkstatt der Universität Kassel. Die Teilnehmer übernehmen dabei Assistenzfunktionen und begleiten die Postproduktion am Computerschnittplatz, bei der Auswahl der Szenen, der jeweiligen Filmmusiken sowie Gestaltung von Titel und Abspann. Am Ende des Workshops, der von montags bis montags durchgeführt 
wird, findet eine Präsentation vor 100 Mitgefangenen sowie Pressevertretern, Gästen von außen und Beamten der Justizvollzugsanstalt statt. Am Ende der Vorführung werden die „Filmemacher" vorgestellt und in einem Feedback diskutieren sie mit den Zuschauern die Sichtweise ihrer Botschaften in ihrem Film. Ziel der Arbeit mit Spielfilm ist es daher, die Alltags- und Gewalterfahrungen der jugendlichen Straftäter, die Kindheits- und Familienerlebnisse sowie die Erfahrungen von Tat und Strafe transparent zu machen, um sie zu hinterfragen und sie mit den anderen Gefangenen $\mathrm{zu}$ kommunizieren. Das Medium Spielfilm dient als hoch motivierendes Medium, mit dem es möglich ist, in der Auseinandersetzung mit dem Thema und sich selbst, seine Erfahrungen und Erlebnisse in der Vergangenheit wieder lebendig werden zu lassen, sich zu spiegeln, Stellung zu beziehen und das eigene Handeln neu zu bewerten. Die Sichtweise der Dinge, eigene Themen, eigene Taten aus einer neuen Perspektive zu sehen und das nicht aus einer anklagenden Situation heraus, sondern aus der Notwendigkeit, eine Geschichte zu erfahren, sie zu verstehen und in den Kontext einer Filmgeschichte $\mathrm{zu}$ stellen. Der Gebrauchs- und Mitteilungswert einer Spielfilmproduktion sagt über die Prozesse und Veränderungen während der gemeinsamen Arbeit jedoch nur wenig aus. So ist es selten, dass die Zuschauer in gleicher Art und Weise wie die Teilnehmer euphorisch und berührt aus einer Präsentation wieder herausgehen. Oft sind es die unmittelbaren Freunde aus den eigenen Wohngruppen in den Strafhafthäusern, die in späteren Diskussionen Veränderungen bemerken.

\section{Forschungsinteresse}

Das Forschungsinteresse zielt von daher nicht nur auf themenorientierte und für die Gefangenen neue Erkenntnisse und Einsichten sozialer Wertehaltung mit verschiedener Tragweite oder darauf, wie Praxis in wissenschaftlich nachvollziehbarer und überprüfbarer Weise weiter entwickelt werden kann. Vielmehr fordert es auch zu neuen Erkenntnissen und der Aneignung eigener veränderter Verhaltensweisen und Fertigkeiten in der Zusammenarbeit mit der Gruppe heraus wie auch zu unkonventionellen Methoden der Erkenntnisgewinnung und Gestaltung der Praxis. Die damit verbundenen wissenschaftlichen Herausforderungen einer überprüfbaren gültigen Theoriebildung bleiben bislang noch verschlossen. Die vorliegenden quantitativen Evaluationen, die Auswertungen der Einzel- und Gruppenreflexionen und Interviews sind unter Validitätskriterien nicht geeignet positive Veränderungsprozesse bei den Gefangenen im Sinne veränderter persönlicher Einstellungen und Wertehaltung quantitativ nachzuweisen. Den- noch ergibt sich eine Reihe von Hinweisen darauf, dass die Mitarbeit im Projekt bei den Teilnehmern Veränderungen im Verhalten und den persönlichen Einstellungen ausgelöst und gefördert hat, Hinweise, die aus unseren subjektiven Verhaltensbeobachtungen der Workshopteilnehmer, aus Einzel- und Gruppengesprächen, Rückmeldungen von Bediensteten der JVA, Sozialarbeiterinnen und Sozialarbeitern aus den Strafhafthäusern und den Auswertungen der Interviews, die Prof. Dauber, Erziehungswissenschaftler an der Universität Kassel, im Rahmen der Prozessberatung in den letzten beiden Jahren durchgeführt hat, gewonnen wurden. „Diese Veränderungen beziehen sich auf neue Möglichkeiten, eigene Gefühle wahrzunehmen und auszudrücken, die eigene Straftat aus anderer Perspektive wahrzunehmen, das eigene Verhalten und die eigenen inneren Beziehungen gegenüber Mitgefangenen, Bediensteten und Mitarbeitern der JVA sowie Mitgliedern der Projektgruppe und der eigenen Herkunftsfamilie zu reflektieren, eigene künftige Handlungsmöglichkeiten realistisch einzuschätzen sowie insbesondere den eigenen Entwicklungsprozess im Rahmen des Projekts, wenigstens ansatzweise, zu reflektieren" (Dauber, in: Nolle/Hildebrandt 2004, S. 145).

Ein weiteres Forschungsinteresse liegt auf der Erforschung und Weiterentwicklung einer medienpädagogischen Theorie- und Praxisbildung, in deren Mittelpunkt die methodische Ausformung und Beschreibung einer prozessorientierten, biografischen Spielfilmarbeit steht. Gegenstand ist das Erproben und Evaluieren praxisrelevanter methodischer und didaktischer Verfahren in pädagogischen Prozessen, um gezielt Spielfilmgenres in Film und Fernsehen zu reflektieren, sich selbst zu reflektieren, die persönlichen Einstellungen $\mathrm{zu}$ sich und seiner sozialen Umwelt neu zu bewerten, zu revidieren und eine themenorientierte Bearbeitung eigener Alltagserfahrung, Einstellung und Wertehaltung zu leisten. Das Interesse orientiert sich an einer differenzierten individuellen Einsicht in die eigene Weiterentwicklung, der Entwicklung der Persönlichkeit und Identität.

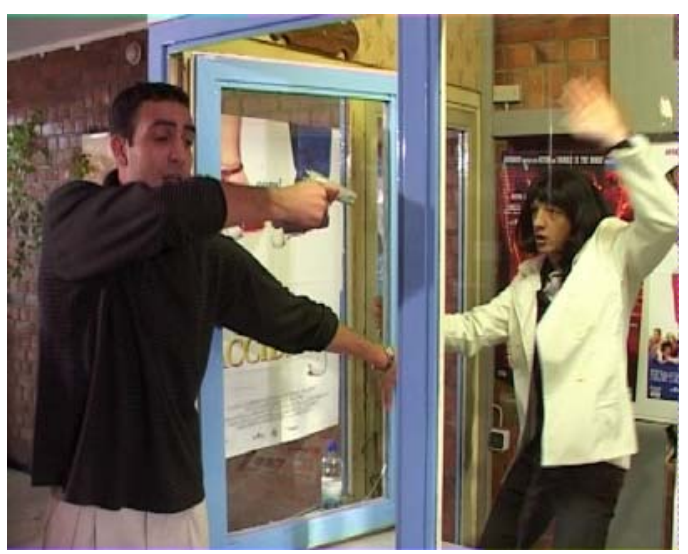


Insgesamt orientiert sich die Spielfilmarbeit an folgenden Kernpunkten:

\section{Das Spielfilm-Genre kennen lernen}

Kids und Jugendliche müssen das Genre Spielfilm kennen lernen, indem sie ihre „eigenen“ Spielfilme über sich selbst machen, lernen, wie man inszeniert und konstruiert, den schwierigen thematischen Schreibprozess an ihren Geschichten und am Drehbuch erfahren, die Pflicht zur Dramaturgie, den technischen Produktionsprozess verstehen und ihre eigenen Schauspielfähigkeiten vor der Kamera spüren, die Pflicht alles abzusprechen und alles zu inszenieren, weil Spielfilm immer aus verschiedenen Perspektiven dreht. Sie lernen, wie wichtig sie dabei sind als Person und in ihrer Rolle, und sie lernen, wie zufriedenen sie sein können, wenn sie ihre Emotionen bei der Präsentation vor vielen Zuschauern erleben und feststellen, wie viel Sinnstiftung Spielfilm mit ihren eigenen Geschichten haben kann.

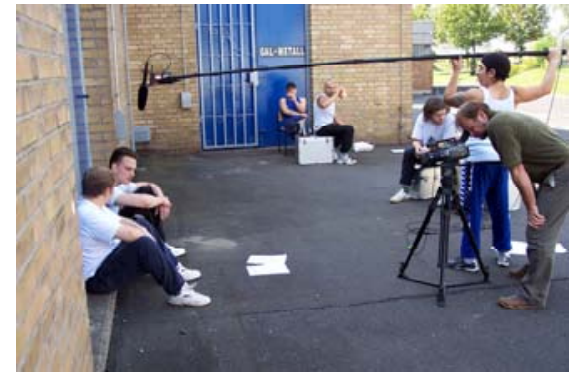

\section{Die Motivation zum Mitmachen ist entscheidend}

Um das zu lernen, muss man die Produktionstechnik nicht selber beherrschen, nicht selber die Kamera führen oder den Film montieren und schneiden. Der Standard von Jugendlichen gegenüber der technisch dramaturgischen Qualität von Fernsehen ist der, den sie fast täglich im Vorabendprogramm der Sender sehen. Das zu imitieren ist ihre Motivation, dafür opfern sie Freizeit und Wochenenden. Sie möchten auch einmal diese Rollen spielen. Es wäre vermessen zu glauben, dass die formale Qualität der Vorabendprogramme, von ungeübten, untrainierten Schülern und Jugendlichen $\mathrm{zu}$ realisieren ist. Spielfilm hat eigene Regeln, hat eine selbständige Sprache, Grammatik und Gestaltungsform, wie jede andere Fremdsprache auch.

Es sollte zum Schluss ein Qualitätsprodukt herauskommen, dass nicht nur in der Peergroup, im Unterricht und zum Jux im Freundeskreis gezeigt wird, manchmal auch das nicht, weil es zu schlecht geworden ist, sondern mit Stolz vor vielen Menschen, Freunden, Eltern, Mitschülern, Lehrern und der Presse. Das ist von der Projektbetreuung zu organisieren. Man ist Stolz auf das, was man geleistet hat, erlebt die eigene Selbstwirksamkeit und präsentiert mit dem Film die textlichen und schauspielerischen Fähigkeiten.

\section{Durch Spielfilmarbeit neue Lernfel- der erschließen}

Es sind vor allem die Bereiche Dramaturgie, Inszenierung, Regie und Schauspielen aber auch der Zwang zu Teamarbeit und Disziplin, die in Spielfilmprojekten gelernt werden. Soziale und empathische Kompetenzen stehen ganz oben in den Lernfeldern. Die Jugendlichen lernen, wie die eigene Geschichte zu integrieren ist in eine gemeinsame Geschichte. Sie lernen, dass alles im Spielfilm inszeniert ist, dass es keine Zufälle gibt, dass z. B. sog. Reality-Shows keine Realität abbilden, sondern inszeniert sind. Sie lernen, dass man selbst tagtäglich viele parallele Geschichten sieht, erlebt oder an ihnen beteiligt ist. Sie lernen, wie man Geschichten in Parallelmontagen konstruiert. Sie erfahren die Bedingung zur Inszenierung, die Abhängigkeit von Dialog, Mimik-Gestik, Bewegung des Körpers, von Maske und Licht, von Bildgestaltung, Rhythmus, Schnittdramaturgie und dem $\mathrm{Zu}-$ sammenspiel auf ein Gesamtwerk, das sie auf der Metaebene immer im Blick haben, dass sie dieses Ziel beeinflussen können durch die Qualität der eigenen Geschichte, die Qualität der Dialoge und die Qualität der schauspielerischen Ausgestaltung der Rolle in ,ihrem“ Film, der Qualität des eigenen sozialen und empathischen Verhaltens. Es dreht sich um „Sie“ selbst; mit Hilfe des Spielfilms als Reflexionsfläche, um das Erkennen und Reflektieren eigener Denk-, Handlungs- und Fühlmuster.

\section{Das Medium Spielfilm als Chance für Reflexion}

In diesem Kontext wird das Medium Spielfilm zum Medium. Die jugendlichen Straftäter müssen acht Tage lang ganz persönlich Stellung beziehen zu ihren kriminellen Taten und Alltagserfahrungen. Beim Diskutieren und beim Spielen vor der Kamera müssen sie mit ihrer ganzen psychischen und physischen Person präsent sein, sonst nimmt man ihnen ihre Erlebnisse, die sie nachspielen und die Authentizität ihrer Rolle im Film nicht ab. Sie müssen sich auf ganz verschiedenen Ebenen und Prozessen (Themendiskussionen, eigene Geschichte, Dialoge und Spielproben vor der Kamera, Diskussion mit den Zuschauern) in Beziehung setzen zu sich, ihrer Gruppe, den Mitarbeitern der MedienWerkstatt mit ihrer Persönlichkeit, ihren Einstellungen, ihren Taten und Strafen.

\section{Emotionale Nähe und soziale Bin- dung fördern}

„Ein wesentliches Ziel bei allen Interaktionen im Schutzraum des Projektes war zu einer Form des Umgangs miteinander zu finden, die nur unzulänglich mit dem Begriff, Vertrautheit' umschrieben werden kann. Ich würde hier lieber eine Formel gebrauchen, die sich aus ,Vertrauen', ,emotionaler Nähe', sozialer Kompetenz, empathischer Fähigkeit' und ,kritischer 
Distanz' zusammensetzt" (Nolle, in: Fuhr/ Dauber 2002, S. 232f.). „Wie FONAGY und Mitarbeiter in ihrer Prison-Health-CareCentre-Studie gezeigt haben, liegt bei 80 $90 \%$ der jugendlichen Straftäter eine Vorgeschichte von Misshandlung vor, die es erschwert bis unmöglich macht, sich in positiver Weise an Individuen und soziale Institutionen $\mathrm{zu}$ binden und entsprechende emotionale und kognitive Steuerungsprozesse zu entwickeln. (Rund ein Viertel der Menschen, die in ihrer Kindheit und Jugend schwer misshandelt wurden, werden später als Straftäter verurteilt)“ (Dauber, in: Nolle/Hildebrandt 2004, S. 145).

Die Spielfilmarbeit zielt darauf ab, emotionale Nähe und soziale Bindung zu fördern. Es geht dabei ,nicht mehr nur um die Herstellung von Bindung an sich, sondern darum, das Kind durch sichere Beziehungen der Art auszustatten, dass es das Verstehen mentaler Zustände im Anderen und im Selbst entwickelt. Das Konzept der Mentalisierung wird so zum zentralen Punkt für das menschliche Funktionieren im sozialen Umfeld" (Dornes 2005, S. 72-81). Vor diesem Hintergrund bekommt das Verständnis für die Zusammenhänge zur Entwicklung sozialer und empathischer Kompetenzen als Schlüssel zur Reflexion des eigenen und des „Anderen“ Denkens und Fühlens und zur Gestaltung des eigenen und des „Anderen“ Konzeptes in sozialen Gemeinschaften eine neue Perspektive. Für Methoden der (Re-)Sozialisation, der Gewaltprävention und -reflexion bedeutet das vor dem Hintergrund eigener Tat-, Alltags- und Kindheitserfahrungen einen wirkungsvollen handlungsorientierten Schwerpunkt auf die Integration von rationaler Einsicht, emotionaler Berührtheit, leiblichem Erleben und Erinnern, sozialer Interaktion, kreativem Handeln sowie Gestaltungsmöglichkeiten in Selbstdarstellungsprozessen zu legen. Die didaktisch-methodische Weiterentwicklung der ,prozessorientierten Spielfilmarbeit" in der Prozess- und Ergebnisforschung muss sich an diesen Erkenntnissen orientieren.

\section{Bilanz von Mai 2001 bis Februar 2005}

In den vergangenen vier Jahren sind mit dieser Methode in drei Gruppen mit insgesamt 28 Teilnehmern bislang 17 Kurzspielfilme entstanden sowie zwei Bücher (siehe unten), die die Gefangenen in den Zeiten zwischen den Workshops geschrieben haben und die in der Presse sowie im Fernsehen und Radio viel Interesse hervorgerufen haben. Von 28 Teilnehmern in vier Jahren, von denen die Mehrzahl an sechs bis acht Workshops teilgenommen hat, sind bis heute noch 13 Teilnehmer in Haft, 15 sind nach zwei Dritteln ihrer Haftstrafe entlassen worden. Von diesen 15 ist bis heute - ein Jahr nach Entlassung - ein Täter rückfällig geworden und sitzt wegen eines Überfalls mit seinen Brüdern wieder in Haft. $85 \%$ Rückfallquote ist sonst die Regel.

Das Projekt wird gefördert im Rahmen des Bundesprogramms „ENTIMON - gemeinsam gegen Gewalt und Rechtsextremismus" durch das Bundesfamilienministerium.

\section{Literatur}

Dornes, Martin: Theorien der Symbolbildung. Psyche I, 59. Jg., 2005, S. 72 - 81.

Nolle, Reinhard, Aktive Medienarbeit: Interkulturelle Dialoge in Projekten handlungsorientierter Pädagogik / kassel university press, Kassel 2001, Dissertation 1999, Überarbeitete u. erweiterte Ausgabe. ISBN 3-933146-74-7, www.upress.uni-kassel.de

Nolle, Reinhard (Hrsg.), Christian B., Mario W., Mark D., Christian M., Michael Z., Wir sagen aus. Band I, Biografische Geschichten und Gedichte jugendlicher Gefangener, kassel university press, Kassel 2002, 159 S., ISBN 3-89958-021-4, www.upress.uni-kassel.de

Nolle, Reinhard Nolle / Hildebrandt, Anke (Hrsg.), Ahmet, Burhan, Christian, David, Deo, Jesse, Marco, Wir sagen aus, Band II, Biografische Geschichten und Interviews jugendlicher Gefangener, kassel university press, Kassel 2004, 196 S., ISBN 3-89958-118-0, www.upress.unikassel.de

Nolle, Reinhard, Spielfilmarbeit mit jugendlichen Strafgefangenen, in: Reinhard Fuhr/ Heinrich Dauber (Hrsg.) Praxisentwicklung im Bildungsbereich - Ein integraler Forschungsansatz, Bad Heilbrunn, 2002, S. $231 \mathrm{ff}$.

\section{Dr. phil. Reinhard Nolle}

(1946) Lehrer/Medienpädagoge Sek. II, Sozialpädagoge, 30 Jahre aktive Medienarbeit in der schulischen-, außerschulischen und universitären Bildung mit Antigewalt- und Suchtpräventionsprojekten, seit 1979 Erziehungs- und Medienwissenschaft an der Universität Kassel FB Erziehungswissenschaft FB Sozialwesen.

Lehr- und Forschungsschwerpunkte:

Methoden aktiver Medienarbeit, Prozessorientierte Spielfilmarbeit, Interkulturelle Medienpädagogik, Methodenforschung zur prozessorientierten Gewalt- und Suchtprävention

Kontakt:

Dr. Reinhard Nolle, Universität Kassel, FB Erziehungswisschenschaft, MedienWerkstatt Arnold Bode Str. $10 \bullet 34109$ Kassel

Tel. 0561/80 42 901, E-Mail:nolle@uni-kassel.de 